\title{
Thermoanalytical, Spectroscopic and Chromatographic Approach to Physicochemical Compatibility Investigation of 5-Aminosalicylates and Folic Acid
}

\author{
(1) Mario-Livio Jeličić, ${ }^{1, *}$ (1) Edvin Brusač, ${ }^{1}$ (1) Stanislav Kurajica, ${ }^{2}$ (1) Matija Cvetnić, ${ }^{3}$ \\ (1) Daniela Amidžić Klarić, ${ }^{1}$ (i) Biljana Nigović, ${ }^{1}$ 미 Ana Mornar ${ }^{1, \#}$
}

\footnotetext{
1 Department of Pharmaceutical Analysis, University of Zagreb, Faculty of Pharmacy and Biochemistry, Zagreb, Croatia

2 Department of Inorganic Chemical Technology and Non-Metals, University of Zagreb, Faculty of Chemical Engineering and Technology, Zagreb, Croatia

3 Department of Analytical Chemistry, University of Zagreb, Faculty of Chemical Engineering and Technology, Zagreb, Croatia

* Corresponding author's e-mail address: mljelicic@pharma.hr

\# Corresponding author's e-mail address: amornar@pharma.hr
}

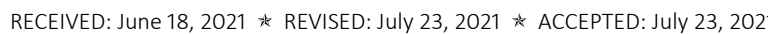

\begin{abstract}
Fixed-dose combinations have shown to be a great alternative to traditional polytherapy; however, development of such formulation requires thorough physicochemical compatibility investigation of active pharmaceutical ingredients to provide a stable, safe and therapeutically effective product. In this work, differential scanning calorimetry, X-ray powder diffraction, isothermal stress testing followed by Fouriertransform infrared spectroscopy and chromatographic analysis as well as dissolution studies were used for physicochemical compatibility investigation of folic acid and balsalazide or olsalazine. Balsalazide and olsalazine as well as their blend were successfully characterised regarding their physicochemical properties using the mentioned techniques. Differential scanning calorimetry gave ambiguous results due to premature degradation of balsalazide. On the contrary, other techniques have implied the absence of any chemical reactions or physical changes in prepared blends. Obtained result imply that folic acid is compatible with both balsalazide and olsalazine which goes in favour of developing proposed fixed-dose combinations.
\end{abstract}

Keywords: balsalazide, olsalazine, folic acid, fixed-dose combination, physicochemical compatibility, FTIR, DSC, XRPD, chromatography

\section{INTRODUCTION}

B ALSALAZIDE (BSZ) and olsalazine (OSZ), anti-inflammatory drugs belonging to the class called 5 -aminosalicylates (5-ASA), have shown to be great alternatives to mesalazine and sulfasalazine-based therapies of inflammatory bowel diseases (IBD). ${ }^{[1,2]}$ Mesalazine is mostly absorbed in the upper parts of the gastrointestinal tract, leaving little to heal the inflammation in the colon, whilst mesalazine carrier sulfapyridine causes adverse reactions in patients whose therapy is based on sulfasalazine. ${ }^{[3,4]}$ BSZ and OSZ are both prodrugs of mesalazine, meaning that active moiety is released upon the metabolism of the drug. BSZ comprises of carrier 4-aminobenzoyl- $\beta$-alanine and mesalazine linked together by an azo bond, while OSZ comprises of two mesalazine molecules connected by azo bond as well. These prodrugs do not tend to be absorbed in the upper part of the gastrointestinal tract and the majority of administered drug reaches the colon where the enzyme azoreductase breaks the azo bond and releases the antiinflammatory moiety, mesalazine, directly to the site of inflammation. ${ }^{[2,5]}$

It is well known that IBD patients suffer from malabsorption of necessary nutrients, such as proteins, fats, carbohydrates, vitamins and minerals. ${ }^{[6-8]}$ Among all mentioned, in this work we are going to focus on the malabsorption of vitamins, more specifically vitamin $B_{9}$, also known as folic acid (FA). FA plays an important role in 
maintaining of normal functioning of the human body and it is mostly ingested through regular nutrition, followed by its absorption in the small intestine. However, as IBD damages parts of the gastrointestinal tract, the small intestine can be affected too, resulting in a reduced area of healthy sidewalls to absorb the FA. Reduced concentration of FA in IBD patients can increase the chance of developing colorectal cancer or megaloblastic anaemia, as well as defects in newborns. ${ }^{\left[{ }^{[-11]}\right.}$ FA deficiency is easily controlled and supplemented by taking a FA oral dosage form during regular therapy of IBD, however, elevated doses of 1-5 mg per day are mandatory compared to the regular intake of $0.4 \mathrm{mg}$ per day. ${ }^{[12]}$

As simple as it sounds that taking only one additional pill during therapy can solve the problem of malabsorbed $\mathrm{FA}$, therapy nonadherence is one of the biggest threats to effective treatment of not only IBD, but also a variety of diseases that are based on polytherapy. ${ }^{[13-15]}$ One of the ways this problem was approached was the development of so-called fixed-dose combinations (FDCs). ${ }^{[16]}$ FDC is a drug product consisting of two, or more, active pharmaceutical ingredients (API) combined in a single formulation, whether in a form of tablet, capsule, or other dosage forms. During the development of such formulation, it has to be ensured that developed FDC has the same therapeutical outcome as it would be if two drugs were taken separately. To secure the safety and efficacy of FDC, during the development the emphasis is on physicochemical stability evaluation of active ingredients when present in the physical blend. ${ }^{[17]}$

Various methods have been used so far for the investigation of drug-drug and drug-excipient compatibility when present in binary blends. Differential scanning calorimetry (DSC) is a thermal technique that offers information about possible physical changes in prepared blends. ${ }^{[18-20]}$ Isothermal stress testing (IST) followed by chromatographic analysis enables monitoring of degradation rate of components present in blends while spectroscopic technique such as Fourier-transform infrared spectroscopy (FTIR) is a non-destructive method for fast screening of samples and offers information regarding physicochemical stability. ${ }^{[18,20,21]} \mathrm{X}$-ray powder diffraction (XRPD) is another non-destructive method used to get an insight into possible changes of polymorphic forms of drugs. ${ }^{[22,23]}$ Dissolution studies followed by chromatographic analysis can offer an insight into the release rate of APIs from prepared formulation as well as their stability in biologically relevant media. ${ }^{20,24]}$

In this work, physicochemical stability of binary blends containing FA and one of the 5-aminosalicylates, BSZ or OSZ, will be examined using the above-mentioned analytical techniques. The aim of such study would open the path to the development of BSZ/FA and OSZ/FA FDCs.

\section{EXPERIMENTAL}

\section{Reagents and Chemicals}

BSZ analytical standard (> 98 \%) (TCl Co., Ltd., Tokyo, Japan), OSZ European Pharmacopoeia Reference Standard (neat) (EDQM, Strasbourg, France) and FA standard (100.6\%) (meets United States Pharmacopoeia (USP) specifications) were obtained from Sigma-Aldrich (St. Louis, MO, USA). Premid $^{\oplus} 750$ mg capsules (Almirall, S.A., Barcelona, Spain) and Dipentum $250 \mathrm{mg}$ capsules (UCB Pharma Ltd., Dunstable, United Kingdom) containing BSZ and OSZ, respectively, were used for the preparation of FDCs. For preparation of samples, buffers, mobile phase and dissolution media following chemicals were used: ultrapure water $(<0.1 \mu \mathrm{S}$ $\mathrm{cm}^{-1}$ ) purified with Series Ultra Clear and Integra Ultra-pure water system (SG Water, Barsbuttel, Germany), methanol HPLC grade solvent ( $\geq 99.9 \%$ ) (Avantor, Deventer, Netherlands), formic acid for HPLC (98 - $100 \%)$ (Merck, Kenilworth, NJ, USA), sodium hydroxide pellets ( $\geq 98.0 \%$ ) (Sigma-Aldrich), di-sodium hydrogen phosphate dihydrate ( $\geq 99.0 \%$ ) and sodium dihydrogen phosphate dihydrate $(\geq$ $99.0 \%$ ) (Kemika, Zagreb, Croatia).

\section{Preparation of Stock and Working Solutions, Blends and Formulations}

For adjustment and validation of chromatographic method, stock solution containing $100 \mu \mathrm{g} \mathrm{mL}^{-1}$ of BSZ, OSZ and FA was prepared by weighing and dissolving each standard in $1 \mathrm{mM} \mathrm{NaOH}$ followed by 1-minute sonication in an ultrasonic bath (Elmasonic XtraTT, Biosan, Riga, Latvia). Working solutions were prepared by diluting the stock solution down to $0.5 \mu \mathrm{g} \mathrm{mL}^{-1}$ to achieve acceptable concentration range of calibration curve for upcoming chromatographic analyses.

Blends containing BSZ and FA standards as well as those containing OSZ and FA standards were prepared in $10: 1,5: 1,2: 1,1: 1,1: 2,1: 5$ and $1: 10$ ratio (the first number represents the share of 5-ASA, second the share of FA) by accurately weighing (using MX5 microbalance scale by Mettler Toledo, Columbus, OH, USA) and mixing both standards using mortar and pestle to secure complete homogenisation.

Proposed BSZ/FA and OSZ/FA FDCs were prepared by emptying and weighing the content of 20 capsules of 5ASA followed by the addition of weighed amount of FA and homogenisation. Capsules were refilled with prepared blends and closed. Prepared FDCs contained $750 \mathrm{mg}$ of BSZ and $1.25 \mathrm{mg}$ of FA or $250 \mathrm{mg}$ of OSZ and $1.25 \mathrm{mg}$ of FA. Homogeneity of prepared blends was examined by chromatographically analysing six samples of each blend, where relative standard deviation (RSD) values of obtained recoveries for each blend did not exceed $0.9 \%$. 


\section{Chromatographic Conditions}

HPLC system Agilent 1100 series (Agilent Technologies, Waldbronn, Germany) coupled with diode-array detector (DAD) was used for analysis of samples. For all chromatographic analyses and data processing, ChemStation software was used. Samples were analysed using the slightly modified version of our previously published method. ${ }^{[25]}$ The linearity range was expanded for BSZ, OSZ and FA for the method to be suitable for the intended use. To confirm the suitability of the modified method, accuracy and precision tests were performed.

\section{Differential Scanning Calorimetry (DSC)}

DSC measurements were performed on Perkin-Elmer Diamond differential scanning calorimeter (Perkin Elmer, Inc., Waltham, MA, USA). Analyses were conducted on BSZ, OSZ and FA standards as well as their blends in the ratio of 10:1, 5:1, 2:1 and 1:1, in favour of 5-ASA. Sample amount of approximately $3 \mathrm{mg}$ was weighed directly into a $50 \mu \mathrm{L}$ aluminium pan and sealed with a pierced aluminium lid. The heating temperature for measurement of BSZ and its blends ranged from 25 to $250{ }^{\circ} \mathrm{C}$ whilst for OSZ and its blends temperature ranged from 25 to $360{ }^{\circ} \mathrm{C}$. Pure nitrogen at a flow rate of $25 \mathrm{~mL} \mathrm{~min}^{-1}$ was used as a purging gas with a heating rate of $10{ }^{\circ} \mathrm{C} \mathrm{min}-1$.

\section{X-ray Powder Diffraction (XRPD)}

Diffractograms of BSZ, OSZ and FA, as well as their 1:1 blend, were obtained on XRD 6000 diffractometer by Shimadzu (Kyoto, Japan) equipped with a wide-focus X-ray tube with CuK $\alpha$ radiation and monochromator, operating at a voltage of $40 \mathrm{kV}$ and current of $30 \mathrm{~mA}$ in a step scan mode between 2 and $42{ }^{\circ} 2 \theta$ with steps of $0.02{ }^{\circ} 2 \theta$ and counting time of $0.6 \mathrm{~s}$.

\section{Isothermal Stress Testing (IST)}

IST was performed on BSZ, OSZ and FA standards as well as on all prepared blends. Samples were spread in a thin layer on a glass dish, visually inspected and placed in an ES-20/60 Orbital Shaker-Incubator by Biosan at an elevated temperature of $50^{\circ} \mathrm{C}$ for 4 weeks. After the defined period samples were removed from the heating chamber and inspected for any visual changes. Samples were prepared for chromatographic analysis by accurately weighing and dissolving $1 \mathrm{mg}$ of heated sample in $10 \mathrm{~mL}$ of $1 \mathrm{mM} \mathrm{NaOH}$ followed by 1-minute sonication.

\section{Fourier Transform Infrared (FTIR) Analysis}

FTIR spectrums of IST stressed samples were measured using Fourier Transform Infrared Spectrophotometer FTIR-8400S by Shimadzu, equipped with PIKE MIRacle ${ }^{\mathrm{TM}}$ universal ATR by PIKE Technologies (Madison, WI, USA).
Each FTIR spectra was obtained as the average of 45 scans, with the resolution of $2 \mathrm{~cm}^{-1}$ in a range from $500-$ $4000 \mathrm{~cm}^{-1}$

For interpretation of obtained FTIR spectra XLSTAT statistical software by Addinsoft (New York, USA) was used. Principal component analysis (PCA) approach followed by cluster analysis (CA) was used on spectral region from 550 - $1800 \mathrm{~cm}^{-1}$ (using Pearson correlation, Euclidean distance measure and Ward's agglomerative clustering). A $9 \times 625$ matrix was created. The number of rows represents the used 5-ASA and FA as well as their blends, whilst spectral data was placed in columns.

\section{In vitro Dissolution Studies}

Testing of drug release from prepared BSZ and OSZ based FDCs was performed on USP 2 dissolution apparatus LDLTA10 (Labtron Equipment Ltd., Fleet, UK) as per USP method for dissolution of BSZ Capsules. ${ }^{[26]}$ Studies were performed in $900 \mathrm{~mL}$ of buffered dissolution media with the $\mathrm{pH}$ set at 6.8 (using FiveEasy pH meter by Mettler Toledo, Columbus, $\mathrm{OH}$, USA) and thermostated at $37.0^{\circ} \mathrm{C}$ with the constant rotation speed of $50 \mathrm{rpm}$. During the dissolution of prepared FDCs, $5 \mathrm{~mL}$ aliquot was drawn from the dissolution vessel every 10 minutes over 30 minutes of dissolution time, filtered through $0.22 \mu \mathrm{m}$ PTFE syringe filter (Obrnuta Faza d.o.o., Pazin, Croatia) directly into HPLC vial and analysed.

\section{RESULTS AND DISCUSSION}

\section{Chromatographic Method Modification}

For all chromatographic measurements used in this study, our previously published in-house method was modified regarding the linearity range. ${ }^{[25]}$ Linearity was broadened to cover the wider concentration range for OSZ, BSZ and FA since the initial method was developed for quality control of the prepared samples, however, this modified version will be applied for monitoring of assay in blends of different ratio. Final linearity of the method was from $0.5-100.0 \mu \mathrm{g}$ $\mathrm{mL}^{-1}$. As none of the chromatographic conditions such as gradient, flow rate, temperature and detection wavelengths were changed, full validation of the method was not necessary, however, verification of the method was done regarding the precision and accuracy, to confirm the applicability of new calibration curves for further calculations. Method parameters such as linearity, accuracy, precision, limit of quantification (LOQ) and limit of detection (LOD) were retested according to the $\mathrm{ICH}$ guideline (Table 1). ${ }^{[27]}$

High linearity was achieved across the desired range with the values of correlation coefficient $(r)$ being higher than 0.9999. The lowest concentration points of calibration 
Table 1. Method verification data

\begin{tabular}{|c|c|c|c|c|c|}
\hline Analyte & Linearity range $\left(\mu \mathrm{g} \mathrm{mL}^{-1}\right)$ & Correlation equation & $r$ & $\operatorname{LOD}\left(\mu \mathrm{g} \mathrm{mL} L^{-1}\right)$ & LOQ $\left(\mu \mathrm{g} \mathrm{mL}^{-1}\right)$ \\
\hline BSZ & $0.5-100.0$ & $y=31.726 x-1.753$ & 0.9999 & 0.14 & 0.5 \\
\hline OSZ & $0.5-100.0$ & $y=37.857 x-0.934$ & 0.9999 & 0.16 & 0.5 \\
\hline \multirow[t]{2}{*}{ FA } & $0.5-100.0$ & $y=30.306 x+0.313$ & 0.9999 & 0.17 & 0.5 \\
\hline & \multicolumn{2}{|c|}{ Precision as RSD (\%) } & \multicolumn{3}{|c|}{ Accuracy as recovery \pm RSD (\%) } \\
\hline Analyte & $\begin{array}{l}\text { Intra-day precision } \\
\qquad(n=6)\end{array}$ & $\begin{array}{c}\text { Inter-day } \\
\text { precision }(n=9)\end{array}$ & $\begin{array}{l}\text { low } \\
(n=3)\end{array}$ & $\begin{array}{l}\text { medium } \\
(n=3)\end{array}$ & $\begin{array}{l}\text { high } \\
(n=3)\end{array}$ \\
\hline BSZ & 1.0 & 1.0 & $100.8 \pm 1.8$ & $101.9 \pm 0.9$ & $101.0 \pm 2.0$ \\
\hline OSZ & 1.3 & 1.2 & $99.7 \pm 1.9$ & $101.7 \pm 1.3$ & $98.0 \pm 2.3$ \\
\hline FA & 1.1 & 1.0 & $99.9 \pm 0.9$ & $101.5 \pm 1.0$ & $98.7 \pm 2.7$ \\
\hline
\end{tabular}

curves $\left(0.5 \mu \mathrm{g} \mathrm{mL^{-1 } )}\right.$ were set as limits of quantification, whilst limits of detection are somewhat lower $(<0.17 \mu \mathrm{g}$ $\mathrm{mL}^{-1}$ ). Precision of the method was retested as intra- and inter-day precision at the concentration of $50 \mu \mathrm{g} \mathrm{mL}^{-1}$. Intra-day precision was calculated as RSD of six individually prepared and analysed samples, whilst inter-day precision was expressed as RSD of three samples prepared and analysed each day, for three consecutive days. Low RSD values for both intra- $(<1.3 \%)$ and inter-day precision $(<1.2 \%)$ imply that the method is precise. The accuracy of the method was tested in triplicate on three concentration levels, lowest $\left(0.5 \mu \mathrm{g} \mathrm{mL} \mathrm{L}^{-1}\right)$, medium $\left(50.0 \mu \mathrm{g} \mathrm{mL}^{-1}\right)$ and highest $\left(100.0 \mu \mathrm{g} \mathrm{mL}^{-1}\right)$ to cover the whole range of calibration curve. Recoveries ranged from 98.0 up to $101.9 \%$ with the RSD (\%) values lower than $2.7 \%$, which implies that the method and calibration curves can be used for accurate recovery of unknown concentrations.

\section{Thermal Analysis Measurements}

Application of thermoanalytical techniques such as DSC in the screening of drug-drug and drug-excipient compatibility has been commonly used in the last decade. The principle of screening is based on comparing exothermic/endothermic peaks present in the thermogram of pure compounds to those obtained by measuring the blends. Results of DSC measurements are present in Figure 1.

BSZ showed characteristic peak with the maximum at $189.5{ }^{\circ} \mathrm{C}$ which occurs due to the dehydration of BSZ dihydrate (Figure 1a). With the further heating of the sample no specific peaks were observed. To verify that BSZ has no specific melting temperature Stuart ${ }^{\mathrm{TM}}$ melting point apparatus SMP3 (Bibby Sterilin Ltd., Staffordshire, UK) was used. It was observed that BSZ started decomposing at the temperatures above $276.0{ }^{\circ} \mathrm{C}$ where the colour of BSZ gradually turned black from starting orange-yellow. Melting of the BSZ was not observed with further heating of the sample up to $360.0^{\circ} \mathrm{C}$. On the other hand, FA showed a characteristic DSC peak at $200.0{ }^{\circ} \mathrm{C}$ which corresponds to its melting point. Precise conclusions cannot be made regarding the BSZ/FA compatibility because BSZ decomposes before it melts, therefore omitting the information regarding the physical changes of BSZ itself. The fact that FA characteristic peak $\left(198.9-201.9^{\circ} \mathrm{C}\right)$ and observed BSZ peak $\left(185.7-190.0^{\circ} \mathrm{C}\right)$ did not shift notably in measured blends might indicate that there is no physical interaction between the two compounds. Any physical change in BSZ or FA would result in a shift of the observed characteristic peaks, indicating that there was a change in crystal structure of the compounds, resulting in different temperature of water release from the structure in case of BSZ or different melting temperature of FA.

.

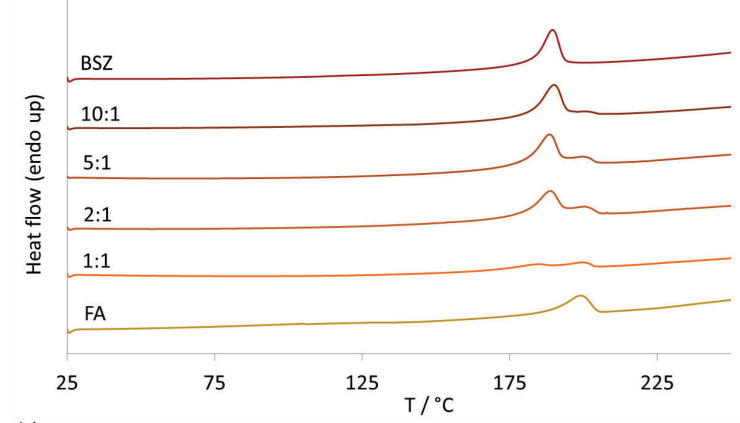

b)

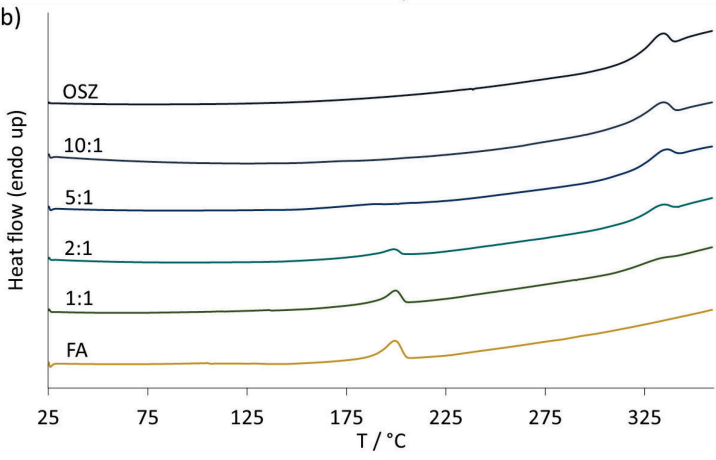

Figure 1. DSC thermograms of a) BSZ, FA and BSZ/FA blends and b) OSZ, FA and OSZ/FA blends. 
OSZ showed an endothermic peak at $333.2{ }^{\circ} \mathrm{C}$ which refers to its melting point (Figure $1 \mathrm{~b}$ ). OSZ/FA blends showed peaks with little to no change in peak temperatures. OSZ peak temperatures ranged from 332.1 to $334.1^{\circ} \mathrm{C}$ whilst FA peak temperatures ranged from 198.9 up to $199.2{ }^{\circ} \mathrm{C}$, showing minor changes in peak temperatures. The broad FA peak is not observed in 5:1 and 10:1 blend, which is mainly due to the low sensitivity of the DSC instrument.

Results presented above could imply that FA is compatible with both BSZ and OSZ, although reaching conclusions solely on results obtained from DSC measurements could mislead the further development process. DSC in combination with other techniques such as XRPD and IST followed by FTIR and chromatographic analysis creates a wider picture where more concise and backed up conclusions can be made.

\section{XRPD results}

XRPD, a non-destructive, non-thermal analytical technique, plays an important role in the drug development process in the preformulation stage for characterisation of crystalline nature of materials used for blend preparation as well as for determining polymorphic nature of APIs. Polymorphic form of API highly impacts its physical properties, therefore it is mandatory to secure their polymorphic stability. APIs that come in crystalline form exhibit unique $\mathrm{X}$-ray diffraction patterns, described with diffraction angles $(2 \theta)$ and intensities of observed peaks. XRPD is a common technique for determining the possible drug-drug and drugexcipients interactions. ${ }^{[28,29]}$ Any physical interactions between the two APIs or API and excipient would result in the change of their characteristic X-ray diffractograms, whether in the form of peak shifting, occurrence of new peaks, or disappearance of existing peaks. Obtained diffractograms show high-intensity sharp peaks characteristic for crystalline APIs (Figure 2).

BSZ shows high-intensity peaks at $2 \theta$ angles of 4.60 , $12.96,15.14,18.14,26.32$ and $26.98^{\circ}$ whilst FA showed
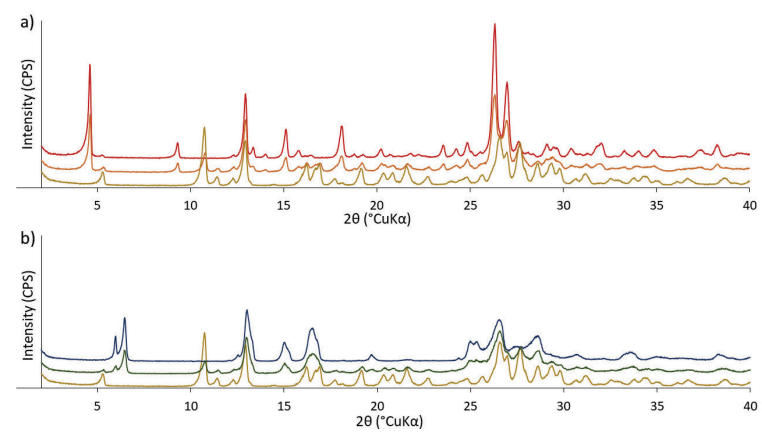

Figure 2. XRPD spectrograms of a) BSZ (red), BSZ/FA (orange) and FA (yellow) and b) OSZ (blue), OSZ/FA (green) and FA (yellow). characteristic peaks at $10.74,12,98,26.66$ and $27.72^{\circ}$. OSZ has shown characteristic peaks at the $2 \theta$ angle of 6.48 , $13.00,16.56$ and $26.56^{\circ}$. X-ray diffractograms of both BSZ/FA and OSZ/FA blends showed all characteristic peaks with no shift in $2 \theta$ values. Moreover, no new peaks were observed with no occurrence of so-called 'halo', which would indicate the presence of amorphous phase. Pearson's correlation was calculated between obtained diffractogram of blend and theoretical diffractogram (calculated as the average of two spectra of pure compounds). Obtained correlation coefficients $(r)$ for BSZ/FA and OSZ/FA blends are 0.99 and 0.98 , respectively. High correlation value between observed and theoretical spectra indicates lack of component interaction.

Obtained results imply that there were no chemical interactions or even changes in the polymorphic forms of both BSZ and OSZ, as well as in the polymorphic form of FA, although they all exhibit polymorphism [30-32], which confirms our assumptions based on DSC screening.

\section{IST followed by chromatographic analysis}

Long term exposure of samples to elevated temperature greatly increases the kinetics of potential reactions and therefore emphasizes the possible drug-drug incompatibility. The best approach to get an insight into the chemical changes of the samples after IST is their quantitative analysis where notable drops of assays imply that components of the binary mixtures decomposed or chemically reacted. Recoveries of OSZ, BSZ, FA, and all their blends are present in Table 2.

Recoveries of all analysed samples do not show a trend in assay changes depending on the share of FA or one of the 5-ASA in the blend. Recoveries of BSZ from BSZ/FA blends are in the range from 98.2 up to $101.3 \%$ with the RSD (\%) values lower than $2.4 \%$, whilst recoveries of FA from those same blends were from 98.3 to $102.5 \%$ with the RSD (\%) values not exceeding $2.2 \%$. Also, by visually inspecting the obtained chromatograms, no new unknown peaks emerged. Recoveries of OSZ from its blends are in the range from 98.3 to $103.7 \%$ with the highest RSD (\%) value of $1.0 \%$. Likewise, recovery of FA from OSZ/FA blends ranged from 97.9 to $103.0 \%$ with the RSD (\%) values lower than $1.7 \%$ showing that no chemical interaction between OSZ and FA occurred. Chromatograms obtained from all analyses showed no new unknown peaks, implying that there was no formation of new products when BSZ or OSZ and $F A$ are present in the same blend, which was confirmed with the peak purity analysis (> 998). Considering the absence of new peaks and recoveries within the blends it can be said that there was no chemical interaction between investigated 5-ASAs and FA. 
Table 2. Chromatographic analysis results of samples conducted to IST

\begin{tabular}{|c|c|c|c|}
\hline Sample & 5-ASA:FA & $\begin{array}{c}\text { Recoveries of 5-ASA } \\
\text { (mean } \pm \text { RSD \%) }\end{array}$ & $\begin{array}{l}\text { Recoveries of FA } \\
(\text { mean } \pm \text { RSD \%) }\end{array}$ \\
\hline \multirow{3}{*}{$\overline{\frac{\alpha}{\alpha}}$} & BSZ & $99.4 \pm 1.9$ & / \\
\hline & osz & $99.9 \pm 1.6$ & I \\
\hline & FA & / & $98.8 \pm 0.6$ \\
\hline \multirow{7}{*}{ 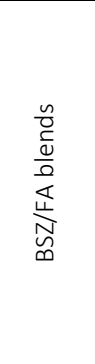 } & $10: 1$ & $99.6 \pm 0.6$ & $99.2 \pm 0.4$ \\
\hline & $5: 1$ & $98.4 \pm 2.7$ & $102.5 \pm 2.2$ \\
\hline & $2: 1$ & $98.8 \pm 1.0$ & $98.3 \pm 1.6$ \\
\hline & 1:1 & $98.2 \pm 2.4$ & $98.4 \pm 1.4$ \\
\hline & $1: 2$ & $100.6 \pm 1.6$ & $98.6 \pm 2.0$ \\
\hline & 1:5 & $99.2 \pm 1.0$ & $100.0 \pm 1.7$ \\
\hline & $1: 10$ & $101.3 \pm 0.7$ & $98.9 \pm 0.8$ \\
\hline \multirow{7}{*}{$\begin{array}{l}\frac{\tilde{y}}{0} \\
\frac{0}{0} \\
\frac{0}{0} \\
\text { 岀 } \\
\tilde{U} \\
0\end{array}$} & $10: 1$ & $99.8 \pm 1.2$ & $97.9 \pm 1.7$ \\
\hline & $5: 1$ & $99.2 \pm 0.2$ & $103.7 \pm 2.4$ \\
\hline & $2: 1$ & $99.1 \pm 2.0$ & $103.0 \pm 0.1$ \\
\hline & $1: 1$ & $99.9 \pm 2.4$ & $99.7 \pm 3.6$ \\
\hline & $1: 2$ & $98.3 \pm 1.0$ & $101.6 \pm 1.9$ \\
\hline & 1:5 & $103.7 \pm 0.6$ & $99.6 \pm 1.9$ \\
\hline & $1: 10$ & $102.6 \pm 0.4$ & $98.7 \pm 1.7$ \\
\hline
\end{tabular}

\section{IST Followed by FTIR and Statistical Analysis}

FTIR is a commonly used method for the investigation of possible physicochemical interactions between two compounds, based on measuring the FTIR spectra of pure compounds as well as their blends, followed by the comparison of obtained spectra. Any changes in the FTIR spectra of measured blends, compared to spectra of pure substances, such as disappearance of characteristic peaks or occurrence of new peaks would indicate that there was a change in the molecules due to their chemical reaction and creation of new products. FTIR spectra obtained from measurements of pure substances and their blends are shown in Figure 3.

BSZ shows characteristic absorption bands at 1639, 1574 and $1484 \mathrm{~cm}^{-1}$ characteristic for $\mathrm{C}=\mathrm{O}, \mathrm{C}=\mathrm{C}$ and $\mathrm{N}=\mathrm{N}$ azo bond, respectively, whilst OSZ shows a wide absorption band at $1583 \mathrm{~cm}^{-1}$ characteristic for aromatic $\mathrm{C}=\mathrm{C}$ bond as well as sharp peak at $1482 \mathrm{~cm}^{-1}$ which is related to the present azo bond. FA absorption bands characteristic for $\mathrm{C}=\mathrm{O}$ and amide $\mathrm{N}-\mathrm{H}$ bonds are observed at 1690 and 1603 $\mathrm{cm}^{-1}$ whilst band at $1482 \mathrm{~cm}^{-1}$ is present due to the pterin ring. ${ }^{[33]}$ Characteristic peaks of BSZ, OSZ and FA can be observed in the prepared blends, with no shifting, disappearance, or occurrence of new peaks.

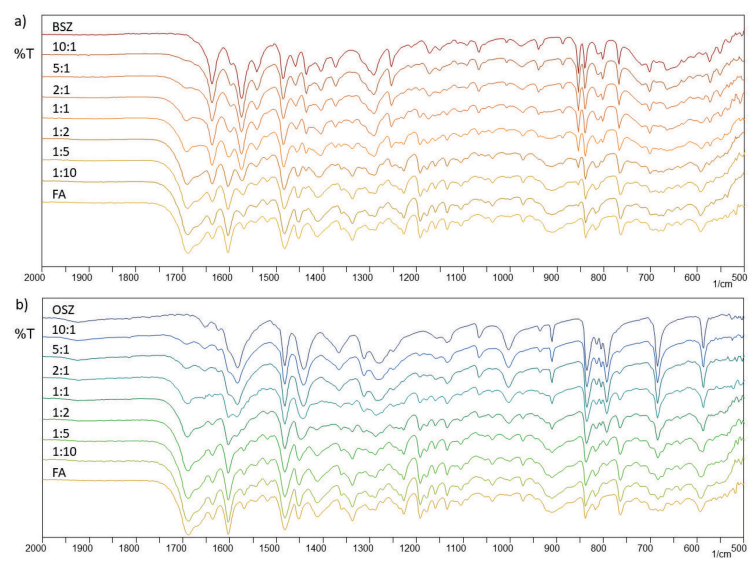

Figure 3. FTIR spectra of a) BSZ, FA and their blends, and b) OSZ, FA and their blends.

Although BSZ, OSZ and FA are not considered large molecules, their complex FTIR spectra are hard to interpret by visual analysis, especially in the case of prepared blends. The interpretation of spectra of physical mixtures may lead to false conclusions due to the overlapping of bands present at similar wavenumbers. Therefore, without going into deeper visual analysis of obtained spectra whereas said above we cannot arrive at an unambiguous conclusion, we turned to a statistical approach with the aim of better interpretation of obtained spectra. Multivariate statistical analysis tools, such as PCA and CA, were already used and are well described in the literature for the interpretation of obtained data. ${ }^{[21,34]}$ The results of PCA and CA calculations are shown in Figure 4.

In the case of BSZ, FA and their blends, the first principal component (PC1) explained $89.63 \%$ of data
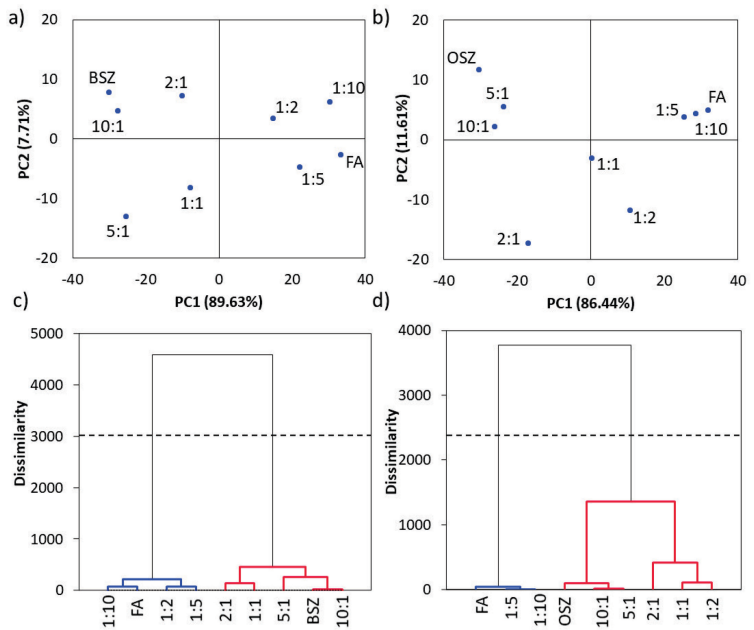

Figure 4. PCA results of a) BSZ, FA and their blends and b) OSZ, FA and their blends as well as accompanying CA results of c) BSZ and d) OSZ based samples. 
variability, whilst the second (PC2) explained only $7.71 \%$ variability. As it can be seen in Figure $4 a$ a, BSZ is placed on the left end with the most negative PC1 value (-30.12), whilst FA with the most positive value (33.47) is placed on the right end of the plot, both representing the values of pure compounds. BSZ/FA blends are positioned in between, where blends with the greater BSZ share are positioned closer to the pure BSZ, whilst blends with a greater share of FA are placed closer to FA. All blends are positioned in the plot in the increasing PC1 values as the BSZ share reduces and FA share in blends increases, with the 1:1 being placed between measured blends. Dendrogram present in Figure 4c reflects the results of the PCA in the form of clusters. Two major clusters were formed with low dissimilarity values within each cluster. First cluster (blue) shows the similarity between FA and blends where FA is in a greater share, whilst the second cluster (red) shows the similarity between BSZ and blends with a greater BSZ share. BSZ/FA 1:1 blend is placed in the cluster with BSZ because of the dominance of its high-intensity peaks in obtained spectra.

In the case of OSZ, similar results were obtained, where PC1 explains $86.44 \%$ data variability whilst PC2 explains $11.61 \%$. As in the case of BSZ, OSZ is placed far to the left with the most negative PC1 value $(-30.27)$ and FA is placed on the opposite side with the most positive PC1 value (31.96) (Figure 4b). All blends are placed between OSZ and FA in the increasing PC1 value as the OSZ share in blends reduces and FA share in blends increases. Dendrogram obtained with CA (Figure 4d) shows 3 clusters with strong linkages. First (blue) consisting of FA and blends with the high FA content, second (red) consists of OSZ and its high content blends whilst the third (red) cluster consists of 2:1, 1:1 and 1:2 blends. The first two clusters reflect the similarity between pure compounds and their dominant blends, where the third cluster shows the similarity between 1:1 blend and blends with slightly higher content of either OSZ or FA. The formation of this cluster is a result of similar absorption bands present in both OSZ and FA spectra at around $1600 \mathrm{~cm}^{-1}$ and in the ranges of $1500-$ $1400 \mathrm{~cm}^{-1}$ and $850-550 \mathrm{~cm}^{-1}$.

Placement of pure compounds and their blends in the PCA plot, as well as the formation of clusters in CA dendrogram, are in favour of BSZ/FA and OSZ/FA compatibility. Any deviation from the obtained order in which blends are positioned would indicate incompatibility between compounds, as well as the formation of clusters with random blends. The absence of new peaks in the obtained FTIR spectra indicate that there was no chemical interaction between compounds as well as there was no disappearance of peaks present in the spectra of pure compounds.

\section{Drug Dissolution Testing}

In vitro dissolution study is often used to display the successful release of the API from its final formulation. In dissolution testing of FDCs, the release of both APIs is monitored during the defined period to ensure that the desired amount of both APIs is dissolved. In addition to the monitoring of drug release, dissolution studies are also used for testing the stability of two APIs when present in the same solution of the $\mathrm{pH}$ similar to those in the gastrointestinal tract. ${ }^{[20,24]}$ Although there is no official method published for dissolution testing of OSZ capsules, BSZ official USP method was applied for both tests. Based on the fact that both BSZ and OSZ have similar $\log k_{w}$ values (3.01 and 3.25, respectively), ${ }^{[25]}$ similar solubility was expected considering the $\mathrm{pH}$ of the used dissolution media (6.8), also, both drugs come in the same dosage forms without modified release and the same excipients present in the formulation. Also, sink conditions are achieved with the defined volume of the dissolution media, considering the high olsalazine solubility in neutral media. ${ }^{[35]}$

Results obtained from the dissolution of twelve prepared BSZ/FA and OSZ/FA FDCs are presented in Figure 5. As per criteria for BSZ Capsules stated in the USP, more than $80 \%$ of the API has to be released from the formulation after the 30-minute test time. As it can be seen
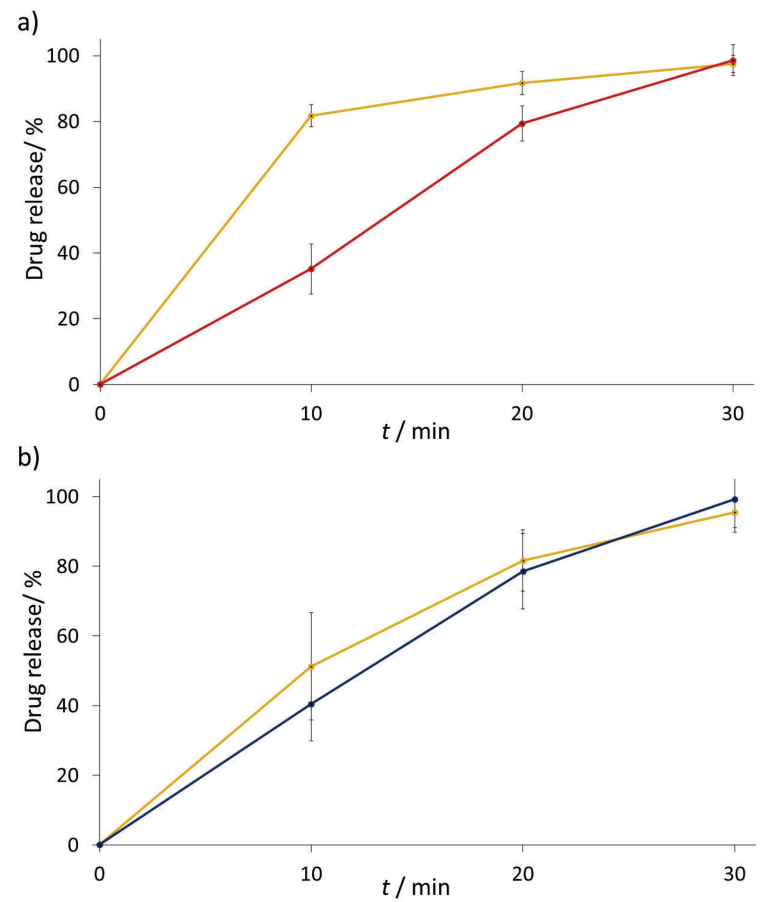

Figure 5. Drug release profiles of a) BSZ (red line) and FA (yellow line) and b) OSZ (blue line) and FA from prepared FDCs $(n=12)$ with the accompanied standard deviation ranges. 
from the results all drug releases are in the range from 91.9 up to $102.0 \%$. FA was as well released almost in total after 30 minutes with the recoveries ranging from 94.9 up to $101.6 \%$. A in the case of OSZ/FA FDCs, OSZ was released in the range from 89.6 up to $102.8 \%$ whilst FA was released from 90.9 up to $103.4 \%$. Faster release of FA from BSZ/FA FDC is due to the greater solubility of BSZ itself compared to the solubility of OSZ as well as the fact that BSZ finished product used for preparation of FDCs contains colloidal anhydrous silica as excipient as well, which serves as an anticaking agent, enabling better solubility of both compounds.

These results indicate that FA can be in the same formulation containing BSZ or OSZ. Considering that BSZ and OSZ capsules contain anhydrous silica and magnesium stearate as excipients, which are commonly used for the preparation of FA finished products, FA stability in their presence is not compromised. The results obtained from dissolution studies imply that FDCs containing FA and BSZ or OSZ are stable, and the liberation of both drugs is satisfactory, although to confirm such conclusions further in vivo studies should be performed.

\section{CONCLUSIONS}

This research was focused on investigating the physicochemical compatibility between FA and two 5-ASAs, OSZ and BSZ. Various analytical techniques were used for mentioned purpose, from thermal such as DSC and IST, to spectroscopic such as FTIR and XRPD as well as chromatographic analysis. Although DSC measurements provided ambiguous results, those obtained with other techniques showed that 5-ASA and FA are physiochemically compatible with no interferences between them. This work opens the path to development of BSZ/FA and OSZ/FA FDCS with the aim of reducing the number of drugs IBD patients need to take during therapy, however further studies are mandatory in regard of developing final formulation as well as bioavailability, safety and efficacy of such product.

Funding Statement. This research was supported by Croatian Science Foundation under grant numbers HRZZ-UIP-201705-3949 and HRZZ-DOK-2018-01-9047.

Conflict of Interest Statement. No potential conflict of interest was reported by the author(s).

\section{REFERENCES}

[1] L.-P. Qian, G.-J. Lin, S.-R. Xu, W.-Q. Ding, Fudan Univ. J. Med. Sci. 2004, 31, 421-424.

[2] J. R. B. Green, A. J. Lobo, C. D. Holdsworth, R. J. Leicester, J. A. Gibson, G. D. Kerr, H. J. F. Hodgson, K. J. Parkins, M. D. Taylor, T. H. E. Abacus, I. Group, Gastroentherology. 1998, 114, 15-22. https://doi.org/10.1016/S0016-5085(98)70627-4
[3] B. Ye, D. van Langenberg, World J. Gastrointest. Pharmacol. Ther. 2015, 6, 137.

[4] S. L. Taffet, K. M. Das, Dig. Dis. Sci. 1983, 28, 833842. https://doi.org/10.1007/BF01296907

[5] A. Ryan, Br. J. Pharmacol. 2017, 174, 2161-2173. https://doi.org/10.1111/bph.13571

[6] Y. Pan, Y. Liu, H. Guo, M. S. Jabir, X. Liu, W. Cui, D. Li, Nutrients. 2017, 9, 1-15. https://doi.org/10.3390/nu9040382

[7] F. K. Ghishan, P. R. Kiela, Gastroenterol. Clin. North Am. 2017, 46, 797-808. https://doi.org/10.1016/j.gtc.2017.08.011

[8] Malnutrition and IBD. Available from: https://www.crohnscolitisfoundation.org/diet-andnutrition/malnutrition-and-ibd. Accessed 20 Apr 2021.

[9] F. Gomollón, J. P. Gisbert, World J. Gastroenterol. 2009, 15, 4659-4665. https://doi.org/10.3748/wjg.15.4659

[10] J. Safi, L. Joyeux, G. E. Chalouhi, J. Pregnancy. 2012, 2012, 295083.

https://doi.org/10.1155/2012/295083

[11] N. Burr, M. Hull, V. Subramanian, J. Clin. Gastroenterol. 2017, 51, 247-253. https://doi.org/10.1097/MCG.0000000000000498

[12] Y. Kubota-Sjogren, K. Harding, P. Irving, C. NelsonPiercy, Br. J. Gen. Pract. 2014, 64, 593-594. https://doi.org/10.3399/bjgp14X682513

[13] W. Chan, A. Chen, D. Tiao, C. Selinger, R. Leong, Intest Res. 2017, 15, 434-445. https://doi.org/10.5217/ir.2017.15.4.434

[14] A. Zelante, A. De Giorgi, R. Borgoni, L. Trevisani, M. Gallerani, Minerva Gastroenterol. Dietol. 2014, 60, 269-274.

[15] J. Lasa, G. Correa, C. Fuxman, L. Garbi, M. E. Linares, P. Lubrano, A. Rausch, M. Toro, M. Yantorno, I. Zubiaurre, L. Peyrin-Biroulet, P. Olivera, Gastroenterol. Res. Pract. 2020, 2020, 4060648. https://doi.org/10.1155/2020/4060648

[16] S. Bangalore, A. Shahane, S. Parkar, F. H. Messerli, Curr. Hypertens. Rep. 2007, 9, 184-189.

[17] Guidelines for registration of fixed-dose combination medicinal products, World Health Organization, WHO Tech. Rep. Ser. (2005).

[18] E. P. Lavor, M. V. M. Navarro, F. D. Freire, C. F. S. Aragão, F. N. Raffin, E. G. Barbosa, T. F. A. De Lima E Moura, J. Therm. Anal. Calorim. 2014, 115, 23032309. https://doi.org/10.1007/s10973-013-3050-2

[19] R. Chadha, S. Bhandari, J. Pharm. Biomed. Anal. 2014, 87, 82-97.

https://doi.org/10.1016/j.jpba.2013.06.016

[20] M.-L. Jeličić, E. Brusač, D. Amidžić Klarić, B. Nigović, S. Keser, A. Mornar, Pharmaceuticals. 2020, 13, 187. https://doi.org/10.3390/ph13080187 
[21] B. Rojek, M. Wesolowski, Vib. Spectrosc. 2016, 86, 190-197.

https://doi.org/10.1016/j.vibspec.2016.07.011

[22] A. Marini, V. Berbenni, M. Pegoretti, G. Bruni, P. Cofrancesco, C. Sinistri, M. Villa, J. Therm. Anal. Calorim. 2003, 73, 547-561. https://doi.org/10.1023/A:1025478129417

[23] H. Seçilmiş Canbay, M. Doğantürk, Celal Bayar Üniversitesi Fen Bilim. Derg. 2019, 15, 401-407. https://doi.org/10.18466/cbayarfbe.613951

[24] V. Petruševska, K. Lasić, A. Mornar, Drug Dev. Ind. Pharm. 2020, 46, 1298-1307. https://doi.org/10.1080/03639045.2020.1788066

[25] M.-L. Jeličić, E. Brusač, D. A. Klarić, B. Nigović, N. Turk, A. Mornar, Sci. Rep. 2020, 10, 1-10. https://doi.org/10.1038/s41598-020-77654-2

[26] The United States Pharmacopeia. The National Formulary, United States Pharmacopoeial Convention, Rockville, MD, USA, 2014.

[27] Validation of analytical procedures: Text and Methodology Q2(R1). Available from: https://database.ich.org/sites/default/files/Q2\%28 R1\%29\%20Guideline.pdf. Accessed 17 Apr 2021.

[28] B. Shah, V. K. Kakumanu, A. K. Bansal, J. Pharm. Sci. 2006, 95, 1641-1665. https://doi.org/10.1002/jps.20644
[29] V. S. Dave, R. V Haware, N. A. Sangave, M. Sayles, M. Popielarczyk, Am. Assoc. Pharm. Sci. 2015, 9-15.

[30] W. Forbes, E. Bortey, L. Johnson, Formulations and Uses of 2-Hydroxy-5-Phenylazobenzoicacid Derivatives for the Treatment of Males, PCT/US20 11/033905, 2011.

[31] M. A. Hofsäss, J. de Souza, N. M. Silva-Barcellos, K. R. Bellavinha, B. Abrahamsson, R. Cristofoletti, D. W. Groot, A. Parr, P. Langguth, J. E. Polli, V. P. Shah, T. Tajiri, M. U. Mehta, J. B. Dressman, J. Pharm. Sci. 2017, 106, 3421-3430. https://doi.org/10.1016/j.xphs.2017.08.007

[32] British Pharmacopoeia, 2020th ed., Medicines and Healthcare Products Regulatory Agency, London, UK, 2020.

[33] Y. Y. He, X. C. Wang, P. K. Jin, B. Zhao, X. Fan, Spectrochim. Acta- Part A Mol. Biomol. Spectrosc. 2009, 72, 876-879.

https://doi.org/10.1016/j.saa.2008.12.021

[34] B. Rojek, M. Wesolowski, B. Suchacz, Spectrochim. Acta- Part A Mol. Biomol. Spectrosc. 2013, 116, 532538. https://doi.org/10.1016/j.saa.2013.07.102

[35] Olsalazine Sodium Catalog No. \$4041. Available from: https://www.selleckchem.com/datasheet/olsalazinesodium-S404101-DataSheet.html. Accessed $20 \mathrm{Apr}$ 2021. 